\title{
EXISTENCE DE SOUS-ESPACES HYPER-INVARIANTS
}

\author{
by K. KELLAY
}

(Received 21 July, 1996)

1. Introduction. Soient $B$ un espace de Banach et $\mathscr{L}(B)$ l'algèbre des opérateurs bornés sur $B$. On dit qu'un sous-espace fermé $E$ de $B$ est invariant pour l'opérateur $T \in \mathscr{L}(B)$ lorsque $T E \subset E$ et qu'il est non trivial si $\{0\} \varsubsetneqq E \varsubsetneqq B$. Le sous-espace $E$ est dit hyper-invariant pour $T$ s'il est invariant pour tout opérateur qui commute avec $T$.

Soit une suite $\left(\tau_{n}\right)_{n \in \mathbb{N}}$ telle que $\tau_{0}=1$ et $\tau_{n} \geq 1$ pour tout $n \geq 0$. On dit que $\left(\tau_{n}\right)_{n \in \mathbb{N}}$ est une suite de Beurling au sens de [3] lorsque

$$
\tau_{n+m} \leq \tau_{n} \tau_{m} \text { pour tout } m, n \in \mathbb{N} \text { et } \sum_{n \in \mathbb{N}} \frac{\log \tau_{n}}{1+n^{2}}<+\infty .
$$

Soit alors $\left(\tau_{n}\right)_{n \in \mathbb{N}}$ une suite croissante de Beurling, soit un opérateur $T \in \mathscr{L}(B)$ tel que $\left\|T^{\prime \prime}\right\|=O\left(\tau_{n}\right)(n \rightarrow+\infty)$. On suppose qu'il existe $x \in B$ tel que

$$
\limsup _{n \rightarrow+\infty} \frac{\left\|T^{n} x\right\|}{\tau_{n}}>0
$$

et une suite $\left(y_{n}\right)_{n \geq 0}$ de $B$ avec $T y_{n+1}=y_{n}(n \geq 0)$ vérifiant la condition (I).

(I): Il existe $C>0$ et $\left(\omega_{n}\right)_{n \in \mathbb{N}}$ une suite croissante de Beurling tels que $\left\|y_{n}\right\| \leq C \omega_{n}$ $(\forall n \in \mathbb{N})$.

Dans le cas où $\tau_{n} \equiv 1(n \in \mathbb{N})$, Beauzamy [4] a montré que la contraction $T$ admet un sous-espace hyper-invariant non trivial (voir aussi [7, Theorem 9.7]). Atzmon a donné une extension de ce résultat $[3$, Theorem 4.5$]$ lorsque

$$
\tilde{\tau}_{n}=\limsup _{m \rightarrow+\infty} \frac{\tau_{n+m}}{\tau_{m}}=O\left(n^{k}\right) \quad(n \rightarrow+\infty)
$$

pour une certaine constante $k$ positive.

Nous parvenons à la même conclusion (Théorème 3.4) lorsque

$$
\tilde{\tau}_{n}=O\left(e^{\varepsilon^{\sqrt{n}}}\right) \quad(n \rightarrow+\infty) \text { pour tout } \varepsilon>0
$$

et lorsque la suite $\left(y_{n}\right)_{n \in \mathbb{N}}$ ci-dessus vérifie la condition (II) au lieu de (I):

$$
\text { (II) }\left\{\begin{array}{c}
\sum_{n \geq 0} \frac{\log ^{+}\left\|y_{n}\right\|}{1+n^{2}}<+\infty \\
\text { il existe } C>0, \text { tel que }\left\|y_{n+1}\right\| \leq C\left\|y_{n}\right\|(\forall n \in \mathbb{N}) .
\end{array}\right.
$$

Remarquons que la condition (II) est plus faible que la condition (I), et c'est cela qui motive ce travail. Notre méthode consiste à faire apparaître des sous-espace invariants liés au prolongement analytique, de même que dans [11]. Ces sous-espaces sont de même nature que ceux mis en évidence dans [3], [4] et [7], mais la considération directe de ces espaces de "vecteurs analytiques" permet d'éviter certaines hypothèses de régularité.

Glasgow Math. J. 40 (1998) 133-141. 
Je tiens à remercier J. Esterle et M. Zarrabi pour les fructueuses discussions que j'ai euex avec eux. Je tiens également à remercier le référé pour ces suggestions.

2. Notations et preliminaires. Un poids $\omega$ est une application de $\mathbb{Z} \rightarrow[1,+\infty[$ telle que $\omega(n+m) \leq \omega(n) \omega(m)$ pour tous $n$ et $m \in \mathbb{Z}$.

Soit $\mathscr{C}(\mathbb{T})$ l'algèbre des fonctions continues sur le cercle unité $\mathbb{T}$ et soit

où

$$
\mathscr{A}_{\omega}(\mathbb{T})=\left\{f \in \mathscr{C}(\mathbb{T}):\|f\|_{\omega}=\sum_{n \in \mathbb{Z}}|\hat{f}(n)| \omega(n)<+\infty\right\},
$$

$$
\hat{f}(n)=\frac{1}{2 \pi} \int_{0}^{2 \pi} f\left(e^{i t}\right) e^{-i n t} d t(n \in \mathbb{Z}) .
$$

$\mathscr{A}_{\omega}(\mathbb{T})$ muni de la norme $\|\cdot\|_{\omega}$ est dite l'algèbre de Beurling associée au poids $\omega$. L'algèbre $\mathscr{A}_{\omega}(\mathbb{T})$ est régulière au sens de [9] si et seulement si

$$
\sum_{n \in \mathbb{Z}} \frac{\log \omega(n)}{1+n^{2}}<+\infty
$$

Le poids $\omega$ est dit alors un poids régulier.

On appelle hyperfonction toute fonction analytique sur $\mathbb{C} \backslash \pi$ telle que

$$
\lim _{|z| \rightarrow \infty} \varphi(z)=0
$$

Les coefficients de Fourier de $\varphi$ sont définis par:

$$
\varphi(z)=\left\{\begin{array}{cc}
\sum_{n \geq 1} \hat{\varphi}(n) z^{n-1} & \text { pour }|z|<1 \\
-\sum_{n \leq 0} \hat{\varphi}(n) z^{n-1} & \text { pour }|z|>1
\end{array}\right.
$$

On désigne par $H D(\mathbb{T})$ l'ensemble de toutes les hyperfonctions. Pour $\varphi \in H D(\mathbb{T})$, le support de $\varphi($ noté $\operatorname{supp} \varphi)$ est le plus petit fermé $E$ de $T$ tel que $\varphi$ se prolonge analytiquement sur $\mathbb{C} \backslash E$.

On identifie le dual de $\mathscr{A}_{\omega}(\mathbb{T})$ à $H D_{\omega}(\mathbb{T})$, où

$$
H D_{\omega}(\mathbb{\mathbb { T }})=\left\{\varphi \in H D(\mathbb{\mathbb { V }}): \sup _{n \in \mathbb{Z}} \frac{|\hat{\varphi}(n)|}{\omega(-n)}<+\infty\right\} ;
$$

la dualité est donnée par:

$$
\langle f, \varphi\rangle=\sum_{n \in \mathbb{Z}} \hat{f}(n) \hat{\varphi}(-n),\left(f \in \mathscr{A}_{\omega}(\mathbb{T}), \varphi \in H D_{\omega}(\mathbb{T})\right) .
$$

Pour $f \in \mathscr{A}_{\omega}(\mathbb{T})$ et $\varphi \in H D_{\omega}(\mathbb{T})$, on définit l'hyperfonction produit $f$. $\varphi$ par ses coefficients de Fourier donnés par la formule:

$$
\widehat{f \cdot \varphi}(n)=\sum_{p \in \mathbb{Z}} \hat{f}(p) \hat{\varphi}(n-p) \text { pour tout } n \in \mathbb{Z} \text {. }
$$

On vérifie que: supp $f . \varphi \subset \operatorname{supp} f \cap \operatorname{supp} \varphi$, où supp $f$ désigne le support fermé de $f$.

Pour $0<a<2 \pi$ et $b>0$, on considère l'arc ouvert

et le domaine

$$
\Gamma_{1}=\{z \in \mathbb{C}:|z|=1 \text { et }-a<\arg z<a\}
$$

$$
\Omega_{1}=\left\{z \in \mathbb{C}: e^{-b}<|z|<e^{b} \text { et }-a<\arg z<a\right\} .
$$


Soit $\rho$ une application continue sur $\left[e^{-b}, e^{b}\right]$ telle que $\rho(r)>0$ si $r \neq 1$ et $\rho(1)=0$. De plus on suppose qu'il existe $\varepsilon>0$ tel que la fonction $\rho$ est décroissante sur $[1-\varepsilon, 1]$ et croissante sur $[1,1+\varepsilon]$. On désigne par $\mathbf{C}_{\rho}\left(\Omega_{1}\right)$ l'espace de Banach des fonctions continues $f: \bar{\Omega}_{1} \backslash \bar{\Gamma}_{1} \rightarrow \mathbb{C}$ telles que la fonction $z \rightarrow \rho(|z|) f(z)$ se prolonge en une fonction continue sur $\bar{\Omega}_{1}$ et nulle sur l'arc fermé $\bar{\Gamma}_{1}$. On munit $\mathbf{C}_{\rho}\left(\Omega_{1}\right)$ de la norme

$$
\|f\|_{\rho}=\sup _{z \in \Omega_{1}}\{\rho(|z|)|f(z)|\} .
$$

On note par $\Omega_{1}^{+}=\Omega_{1} \cap \mathbb{D}$ et $\Omega_{1}^{-}=\Omega_{1} \cap(\mathbb{C} \backslash \overline{\mathbb{D}})$, où $\overline{\mathbb{D}}$ est l'adhérence du disque unité $\mathbb{D}$. On définit les ensembles suivants:

$$
\mathbf{A}_{\rho}\left(\Omega_{1}^{ \pm}\right)=\left\{f: f \in \mathbf{C}_{p}\left(\Omega_{1}\right), f \text { est holomorphe } \operatorname{sur} \Omega_{1}^{+} \cup \Omega_{1}^{-}\right\}
$$

$\mathbf{A}_{\rho}\left(\Omega_{1}\right)=\left\{f: f \in \mathbf{A}_{\rho}\left(\Omega_{1}^{ \pm}\right), f\right.$ a un prolongement analytique à travers l'arc $\left.\Gamma_{1}\right\}$.

THÉORÈME 2.1 (Beurling [5]). Si

$$
\int_{-b}^{0} \log \log \frac{1}{\rho\left(e^{-\sigma}\right)} d \sigma+\int_{0}^{b} \log \log \frac{1}{\rho\left(e^{-\sigma}\right)} d \sigma<+\infty,
$$

alors $\mathbf{A}_{\rho}\left(\Omega_{1}\right)$ est un ensemble fermé de $\mathbf{A}_{\rho}\left(\Omega_{1}^{ \pm}\right)$pour la norme $\|\cdot\|_{\rho}$.

3. Extension du théorème de Beauzamy-Atzmon. On a besoin des lemmes suivants.

Lemme 3.1. Soit $\left(\tau_{n}\right)_{n \in \mathbb{N}}$ une suite croissante de Beurling. Il existe alors une suite de Beurling croissante $\left(\beta_{n}\right)_{n \in \mathbb{N}}$ telle que la suite $\left(\frac{\log \beta_{n}}{n}\right)_{n \geq 1}$ est décroissante et telle que pour
tout $n \in \mathbb{N}$ on a $\log \tau_{n} \leq \log \beta_{n} \leq 4 \log \tau_{n}$.

Preuve. Puisque $\tau_{n+m} \leq \tau_{n} \tau_{m}(\forall n, m \in \mathbb{N})$, il est facile de voir que la suite $\left(\frac{\log \tau_{2^{p}}}{2^{p}}\right)_{p \in \mathbb{N}}$ est décroissante. On pose alors

$$
\gamma_{n}=\frac{n-2^{p}}{2^{p+1}-2^{p}} \frac{\log \tau_{2^{p+1}}}{2^{p+1}}+\frac{2^{p+1}-n}{2^{p+1}-2^{p}} \frac{\log \tau_{2^{p}}}{2^{p}} \text { si } n \in\left[2^{p}, 2^{p+1}\right] .
$$

La suite $\left(\gamma_{n}\right)_{n \geq 1}$ ainsi construite est décroissante. Comme la suite $\left(\tau_{n}\right)_{n \in \mathbb{N}}$ est croissante, on a pour tout $n \in\left[2^{p}, 2^{p+1}\right]$

$$
\begin{gathered}
\gamma_{n} \leq \frac{\log \tau_{2^{p}}}{2^{p}} \leq \frac{\log \tau_{n}}{n} \frac{n}{2^{p}} \leq 2 \frac{\log \tau_{n}}{n}, \\
\gamma_{n} \geq \frac{\log \tau_{2^{p+1}}}{2^{p+1}} \geq \frac{\log \tau_{n}}{n} \frac{n}{2^{p+1}} \geq \frac{1}{2} \frac{\log \tau_{n}}{n} .
\end{gathered}
$$

Donc

$$
\log \tau_{n} \leq 2 n \gamma_{n} \leq 4 \log \tau_{n} \quad \text { pour tout } n \geq 1
$$

On pose $\beta_{n}=\exp \left(\sup _{1 \leq k \leq n} 2 k \gamma_{k}\right)$ pour $n \geq 1$ et $\beta_{0}=1$. Il est clair que la suite $\left(\beta_{n}\right)_{n \in \mathbb{N}}$ est 
croissante, $\beta_{n+m} \leq \beta_{n} \beta_{m} \quad(\forall n, m \in \mathbb{N})$ et que $\log \tau(n) \leq \log \beta(n) \leq 4 \log \tau(n)$. Donc $\left(\beta_{n}\right)_{n \in \mathbb{N}}$ est une suite croissante de Beurling. De plus on a:

$$
\frac{\log \beta_{n+1}}{n+1}=\max \left\{\frac{1}{n+1} \sup _{1 \leq k \leq n} 2 k \gamma_{k}, \frac{2(n+1) \gamma_{n+1}}{n+1}\right\},
$$

comme $\gamma_{n+1} \leq \gamma_{n}$ et $\frac{1}{n+1} \leq \frac{1}{n}$ alors

$$
\frac{\log \beta_{n+1}}{n+1} \leq \max \left\{\frac{1}{n} \sup _{1 \leq k \leq n} 2 k \gamma_{k}, \frac{2 n \gamma_{n}}{n}\right\}=\frac{\log \beta_{n}}{n} .
$$

Ceci termine la preuve du lemme.

Le lemme qui suit est implicitement contenu dans la preuve du lemme 1 [11].

Lemme 3.2. Soit $\left(\beta_{n}\right)_{n \in \mathbb{N}}$ une suite croissante telle que $\beta_{n} \geq 1$ pour tout $n \in \mathbb{N}$ et la suite $\left(\frac{\log \beta_{n}}{n}\right)_{n \geq 1}$ est décroissante et tend vers 0 . Si $\sum_{0}^{\infty} \frac{\log \beta_{n}}{1+n^{2}}<+\infty$, alors

$$
\int_{1}^{e^{-1}} \log \log \left(\sum_{0}^{+\infty} \beta_{n} e^{-n \omega}\right)<+\infty
$$

Le lemme suivant est une variante du théorème de Katznelson-Tzafriri version Allan-Ransford [1] (voir aussi [10]). On désignera par $\sigma(T)$ le spectre de $T$.

Lemme 3.3. Soit $\left(\tau_{n}\right)_{n \in \mathbb{N}}$ une suite croissante de Beurling avec

$$
\tilde{\tau}_{n}:=\limsup _{m \rightarrow+\infty} \frac{\tau_{n+m}}{\tau_{m}}=O\left(e^{r \cdot \sqrt{n}}\right)(n \rightarrow+\infty) \text { pour tout } \varepsilon>0 .
$$

Soit $T$ un opérateur sur un espace de Banach $B$ tel que $\left\|T^{n}\right\|=O\left(\tau_{n}\right)(n \rightarrow+\infty)$. Si $\sigma(T) \cap \mathbb{T}=\{1\}$ alors

$$
\lim _{n \rightarrow+\infty} \frac{1}{\tau_{n}}\left\|T^{n}(T-I)\right\|=0 .
$$

Preuve. On utilise essentiellement la même démonstration que celle de [10] et on conclut avec [2, Theorem 1.1].

On est alors en mesure de démontrer le théorème suivant.

THÉORÈme 3.4. Soit $\left(\tau_{n}\right)_{n \in \mathbb{N}}$ une suite croissante de Beurling avec

$$
\tilde{\tau}_{n}=O\left(e^{r^{\sqrt{n}}}\right)(n \rightarrow+\infty) \text { pour tout } \varepsilon>0 .
$$

Soit $T$ un opérateur de $\mathscr{L}(B)$, non égal à un multiple de l'identité tel que

$$
\left\|T^{\prime \prime}\right\|=O\left(\tau_{n}\right)(n \rightarrow+\infty) .
$$

On suppose qu'il existe $y \in B$ avec

$$
\limsup _{n \rightarrow+\infty} \frac{\left\|T^{n} y\right\|}{\tau_{n}}>0
$$


et une suite $\left(y_{n}\right)_{n \geq 0}$ de $B$ telle que

$$
\begin{gathered}
T y_{n+1}=y_{n} \text { pour tout } n \geq 0, \\
\sum_{n \geq 0} \frac{\log ^{+}\left\|y_{n}\right\|}{1+n^{2}}<+\infty \\
\text { et }\left\|y_{n+1}\right\| \leq C\left\|y_{n}\right\|(\forall n \in \mathbb{N}), \text { où } C \text { est une constante strictement positive. }
\end{gathered}
$$

Alors $T$ possède un sous-espace hyper-invariant non trivial.

Preuve. Puisque nous voulons montrer l'existence d'un sous-espace hyper-invariant pour $T$, on peut supposer, sans restriction de généralité, que:

(i) L'opérateur $T$ est injectif et d'image dense, faute de quoi $\operatorname{Ker} T$ on l'adhérence de Im $T$ seront des sous-espaces hyper-invariants non triviaux.

(ii) $\frac{\left\|T^{\prime \prime} x\right\|}{\tau_{n}} \nrightarrow 0$ quand $n \rightarrow+\infty$ pour tout $x \in B \backslash\{0\}$, sinon l'ensemble

$$
\left\{x \in B, \frac{\left\|T^{n} x\right\|}{\tau_{n}} \underset{n \rightarrow+\infty}{\longrightarrow} 0\right\}
$$

est un sous-espace hyper-invariant non trivial.

Notons que $\sigma(T) \cap \mathbb{T}$ contient nécessairement plus de deux points. En effet: d'une part si $\sigma(T) \subset \mathbb{D}$, on a $\lim _{n \rightarrow+\infty}\left\|T^{n}\right\|^{1 / n}=\sup _{\mu \in \sigma(T)}|\mu|<1$, et donc $\left\|T^{n} x\right\| \underset{n \rightarrow+\infty}{\longrightarrow} 0$ pour tout $x \in B$, ce qui contredit (3).

D'autre part si $\sigma(T) \cap T=\{\lambda\}$, il résulte du lemme 3.3 que pour tout $x \in B$

$$
\frac{1}{\tau_{n}}\left\|T^{n}(\lambda I-T) x\right\| \underset{n \rightarrow+\infty}{\longrightarrow} 0,
$$

et d'après (ii), on a $(\lambda I-T) x=0$. Ceci implique que $T$ est multiple de l'identité, ce qui est en contradiction avec les hypothèses du théorème.

Considérons maintenant la semi-norme définie par

$$
\|x\|=\limsup _{n \rightarrow+\infty} \frac{\left\|T^{n} x\right\|}{\tau_{n}} \text { pour } x \in B .
$$

En tenant compte de (ii), on a Ker $\|.\| \|=\{0\}$; soit alors $\tilde{B}$ la complétion de $B$ munie de la norme \|\|$. \|$. Remarquons qu'il existe $k>0$ tel que $\|x\| \leq k\|x\|$ pour tout $x \in B$, de sorte que l'injection $B \rightarrow \widetilde{B}$ est continue. D'autre par si $R: B \rightarrow B$ est continue et commute avec $T$ on a $\|R x\| \leq\|R\|\|x\|$, et $R$ admet un prolongement continu $\tilde{R}: \tilde{B} \rightarrow \tilde{B}$ tel que $\|\tilde{R}\|=\sup _{\|u\| \leq 1}\|\tilde{R} u\| \leq\|R\|$. En particulier $T$ admet un prolongement continu $\tilde{T}: \tilde{B} \rightarrow \tilde{B}$. L'opérateur $\tilde{T}$ est inversible et $\sigma(\tilde{T}) \subset \sigma(T) \cap \mathbb{T}$, (voir par exemple [10]). On a pour tout $x \in B$

Donc

$$
\left\|T^{n} x\right\|=\limsup _{m \rightarrow+\infty} \frac{\left\|T^{n+m} x\right\|}{\tau_{n+m}} \frac{\tau_{n+m}}{\tau_{m}} \leq \tilde{\tau}_{n}\|x\| \text { et }\|T x\| \geq\|x\| .
$$

$$
\left\|\tilde{T}^{n}\right\|=O\left(e^{\varepsilon^{\sqrt{n}}}\right)(n \rightarrow+\infty) \text { et }\|\| \tilde{T}^{-1} \| \leq 1
$$


Le spectre de $\tilde{T}$ contient au moins deux points car dans le cas contraire, si on note $\lambda$ l'unique élément de $\sigma(\tilde{T})$, il résulte de [2, Theorem 1.1] que $\tilde{T}=\lambda \tilde{I}$, où $\tilde{I}$ est l'opérateur identité sur $\tilde{B}$, ce qui contredit le fait que $T \neq \lambda I$.

Soient alors $\lambda_{1}$ et $\lambda_{2} \in \sigma(\tilde{T}) \subset \sigma(T) \cap \mathbb{T}, \lambda_{1} \neq \lambda_{2}$. On va supposer sans perte de généralité que $\lambda_{1}=1$. Soit l'arc ouvert

$$
\Gamma_{1}=\{z \in \mathbb{C}:|z|=1 \text { et }-a<\arg z<a\},
$$

pour une certaine constante $a>0$ de telle sorte que $\lambda_{2} \notin \bar{\Gamma}_{1}$.

On considère l'ensemble

$$
\tilde{E}=\left\{\tilde{x} \in \tilde{B}: z \rightarrow(\tilde{T}-z \tilde{I})^{-1} \tilde{x} \text { a un prolongement analytique à travers l'arc } \Gamma_{1}\right\} .
$$

Nous allons montrer en premier que $E=\tilde{E} \cap B$ est un sous-espace hyper-invariant pour $T$ distinct de $B$. Soit $R$ un opérateur borné sur $B$ et soit $\tilde{R}$ son prolongement à $\tilde{B}$ défini plus haut. Il est clair que $\tilde{E}$ est un sous-espace invariant pour $\tilde{R}$ et par conséquent $E$ est invariant par $R$.

Montrons maintenant que $E$ est un sous-espace formé de $B$ pour la norme $\|\cdot\|$. Puisque l'injection $B \rightarrow \tilde{B}$ est continue, il suffit de montrer que $\tilde{E}$ est un fermé de $\tilde{B}$ pour la norme $\|\mid\| \cdot$.

Notons par $\tilde{B}^{*}$ le dual de $(\tilde{B},\|\cdot\| \|)$. Soit $\left(\tilde{x}_{n}\right)_{n \geq 0}$ une suite de $\tilde{E}$ qui converge vers $\tilde{x} \in \tilde{B}$ et soit $l \in \tilde{B}^{*}$. On pose

et

$$
\varphi_{n}(z)=\left\langle(\tilde{T}-z \tilde{I})^{-1} \tilde{x}_{n}, l\right\rangle \text { et } \varphi(z)=\left\langle(\tilde{T}-z \tilde{I})^{-1} \tilde{x}, l\right\rangle(z \notin \sigma(\tilde{T})),
$$

$$
\rho(r)= \begin{cases}(1-r)^{2} & \text { si } r \leq 1 \\ (r-1)\left(\sum_{n \leq 0} \tau_{-n} r^{n-1}\right)^{-1} & \text { si } r>1 .\end{cases}
$$

Il est clair que l'application $\rho$ est continue sur $\left[e^{-b}, e^{b}\right]$ pour tout $b>0$, décroissante sur $\left[e^{-b}, 1\right]$ et croissante sur $\left[1, e^{b}\right]$. On fixe $b$ avec $1<e^{b}<\frac{e+1}{e}$. Notons que

$$
(\tilde{T}-z \tilde{I})^{-1}=\left\{\begin{array}{cc}
\sum_{n \geq 1} \tilde{T}^{-n} z^{n-1} & \text { pour }|z|<1 \\
-\sum_{n \leq 0} \tilde{T}^{-n} z^{n-1} & \text { pour }|z|>1
\end{array}\right.
$$

et puisque $\left\|\tilde{T}^{-1}\right\|\|\leq 1,\| \tilde{T}^{n}\|\| \leq\left\|T^{n}\right\| \leq k \tau(n)$ pour tout $n \geq 0$ alors

$$
\left\|(\tilde{T}-z \tilde{I})^{-1}\right\| \leq k \frac{|1-| z||}{\rho(|z|)} \text { pour } z \in \mathbb{C} \backslash \mathbb{T} .
$$

Donc $\varphi \in \mathbf{A}_{\rho}\left(\Omega_{1}^{ \pm}\right)$et puisque $\tilde{x}_{n} \in \tilde{E}, \varphi_{n} \in \mathbf{A}_{\rho}\left(\Omega_{1}\right)$, où

$$
\Omega_{1}=\left\{z \in \mathbb{C}: e^{-b}<|z|<e^{b} \text { et } \frac{z}{|z|} \in \Gamma_{1}\right\}, \quad \Omega_{1}^{+}=\Omega_{1} \cap \mathbb{D} \text { et } \Omega_{1}^{-}=\Omega_{1} \cap(\mathbb{C} \backslash \overline{\mathbb{D}}) .
$$


En outre pour $z \in \Omega_{1}, z \notin \Gamma_{1}$ on a:

$$
\begin{aligned}
\rho(|z|)\left|\varphi_{n}(z)-\varphi(z)\right| & \leq \rho(|z|)\left\|(\tilde{T}-z \tilde{I})^{-1}\right\| \mid\left\|\tilde{x}_{n}-\tilde{x}\right\|\|\| l \| \\
& \leq k(|1-| z||)\left\|\tilde{x}_{n}-\tilde{x}\right\|\|\| l \| .
\end{aligned}
$$

Donc

$$
\left\|\varphi_{n}-\varphi\right\|_{\rho}=\sup _{z \in \Omega_{1}}\left\{\rho(|z|)\left|\varphi_{n}(z)-\varphi(z)\right|\right\} \underset{n \rightarrow+\infty}{\longrightarrow} 0
$$

Puisque la suite $\left(\tau_{n}\right)_{n \in \mathbb{N}}$ est une suite de Beurling croissante, on sait d'après le lemme 3.1 qu'il existe une suite $\left(\beta_{n}\right)_{n \in \mathbb{N}}$ de Beurling croissante telle que la suite $\left(\frac{\log \beta_{n}}{n}\right)_{n \geq 1}$ est décroissante et telle que $\tau_{n} \leq \beta_{n}$ pour tout $n \in \mathbb{N}$; il découle alors du lemme 3.2 que

$$
\int_{0}^{b} \log \log \left(\sum_{n \geq 0} \tau_{n} e^{-n \sigma}\right) d \sigma<+\infty
$$

on conclut alors que

$$
\int_{-b}^{0} \log \log \frac{1}{\rho\left(e^{-\sigma}\right)} d \sigma+\int_{0}^{b} \log \log \frac{1}{\rho\left(e^{-\sigma}\right)} d \sigma<+\infty .
$$

Donc, par le théorème 2.1 de Beurling, $\mathbf{A}_{\rho}\left(\Omega_{1}\right)$ est fermé dans $\mathbf{A}_{\rho}\left(\Omega_{1}^{ \pm}\right)$pour la norme $\left\|_{0}\right\|_{\rho}$ et puisque $\lim _{n \rightarrow+\infty}\left\|\varphi_{n}-\varphi\right\|_{\rho}=0$, on conclut que $\varphi \in \mathbf{A}_{\rho}\left(\Omega_{1}\right)$. Ainsi pour tout élément $l \in \tilde{B}^{*}$, la fonction $z \rightarrow\left\langle(\tilde{T}-z \tilde{I})^{-1} \tilde{x}, l\right\rangle$ a un prolongement analytique à travers l'arc $\Gamma_{1}$, ce que entraîne que la fonction $z \rightarrow(\tilde{T}-z \tilde{I})^{-1} \tilde{x}$ est prolongeable analytiquement à travers l'arc $\Gamma_{1}$, (voir [3, Lemme 2.4]). Par conséquent $\tilde{x} \in \tilde{E}$ et donc $\tilde{E}$ est un fermé de $\tilde{B}$ pour la norme \|\| . $\|$. De là, on déduit que $E$ est un fermé de $B$ pour la norme $\|$.$\| .$

Nous allons montrer maintenant que $E \neq B$. Rappelons qu'on a supposé que $1 \in \sigma(\tilde{T})$. Il découle du théorème de Helson [8, Theorem 3] qu'il existe un vecteur $\tilde{v} \in \tilde{B}$ tel que la fonction $z \rightarrow(\tilde{T}-z \tilde{I})^{-1} \tilde{v}$ n'a de prolongement analytique en aucun voisinage de 1. Donc $\tilde{v} \notin \tilde{E}$ et par conséquent $\tilde{E} \varsubsetneqq \tilde{B}$. Puisque $B$ est dense dans $\tilde{B}$ pour la norme $\|\mid \cdot\|$ et $E \subset \tilde{E}$, on en déduit que $E \varsubsetneqq B$.

Si $E \neq\{0\}$, alors d'après ce qui précède, $E$ est évidemment un sous espace hyper-invariant non trivial pour $T$.

Supposons maintenant la contraire, soit $E=\{0\}$. Considérons la suite suivante:

$$
\omega_{n}= \begin{cases}\tau_{n} & \text { pour } n \geq 1 \\ \max \left(\left\|y_{-n}\right\|, 1\right) & \text { pour } n \leq 0\end{cases}
$$

Pour tout $n \in \mathbb{Z}$ on a $C^{-1} \omega_{n} \leq \omega_{n+1} \leq C \omega_{n}$ pour une certaine constante $C>0$ et

$$
\sum_{n \in \mathbb{Z}} \frac{\log \omega_{n}}{1+n^{2}}<+\infty
$$


Soit $0<\beta<2 \pi$ tel que $e^{i \beta}=\lambda_{2} \in \sigma(\tilde{T})$. Soient $\varepsilon>0$ et l'arc

$$
\Gamma_{2}=\{z \in \mathbb{C}:|z|=1, \beta-\varepsilon<\arg z<\beta+\varepsilon\} .
$$

Il résulte du théorème de Beurling-Malliavin [6, Theorem 1], version discrète (voir [3, Lemme 2.1]), qu'il existe une fonction non nulle, $f \in \mathscr{C}(\mathbb{T})$ telle que le support de $f$ est contenu dans $\Gamma_{2}$ et

$$
\sum_{n \in \mathbb{Z}}|\hat{f}(n)| \omega_{n}<+\infty
$$

En prenant $\varepsilon$ assez petit et un utilisant une rotation si nécessaire, on peut supposer que $f\left(\lambda_{2}\right) \neq 0$ et $f \equiv 0$ sur l'arc fermé $\bar{\Gamma}_{1}$.

Posons

$$
\bar{\omega}(n)= \begin{cases}\tau_{n} & \text { pour } n \geq 1 \\ 1 & \text { pour } n \leq 0\end{cases}
$$

Puisque $\left(\tau_{n}\right)_{n \in \mathbb{N}}$ est une suite croissante et $\tau_{n+m} \leq \tau_{n} \tau_{m}(n, m \in \mathbb{N})$ alors $\bar{\omega}$ définit un poids sur $\mathbb{Z}$. Il est clair que $f \in \mathscr{A}_{\bar{\omega}}(\mathbb{T})$. On pose $f(T)=\sum_{n \in \mathbb{Z}} \hat{f}(n) \tilde{T}^{n} ; f(\tilde{T})$ est bien définie comme opérateur borné sur $(\tilde{B},\|\cdot\|)$ et $f(\tilde{T}) \neq 0$ puisque $f(\sigma(\tilde{T})) \subset \sigma(f(\tilde{T}))$ et $f\left(\lambda_{2}\right) \neq 0$.

Soit $\left(x_{n}\right)_{n \in \mathbb{Z}}$ la suite définie par:

$$
x_{n}= \begin{cases}y_{-n} & \text { pour } n \leq 0 \\ T^{n} y_{0} & \text { pour } n \geq 1\end{cases}
$$

On a pour tout $n \leq 0, \tilde{T}^{-n} x_{n}=y_{0}$ et donc $\tilde{T}^{n} y_{0}=x_{n}$. Donc pour tout $N \geq 0$,

$$
\sum_{|n| \leq N} \hat{f}(n) x_{n}=\sum_{|n| \leq N} \hat{f}(n) \tilde{T}^{n} y_{0}
$$

Puisque $f(\tilde{T})=\sum_{n \in \mathbb{Z}} \hat{f}(n) \tilde{T}^{n}$ converge en norme dans $\mathscr{L}(\tilde{B})$ on a évidemment

$$
\left.\left\|\sum_{|n| \leq N} \hat{f}(n) \tilde{T}^{n} y_{0}-f(\tilde{T}) y_{0}\right\|\right|_{N \rightarrow+\infty} \underset{\longrightarrow}{\longrightarrow} 0 .
$$

D'autre part $\sum_{n \in \mathbb{Z}} \hat{f}(n) x_{n}$ converge en norme dans $(B,\|\|$.$) et donc on a$

$$
\left\|\sum_{|n| \leq N} \hat{f}(n) \tilde{T}^{n} y_{0}-\sum_{n \in \mathbb{Z}} \hat{f}(n) x_{n}\right\| \underset{N \rightarrow+x}{\longrightarrow} 0
$$

Mais comme $\|x\| \leq k\|x\|$ pour tout $x \in B$ alors $f(\tilde{T}) y_{0}=\sum_{n \in \mathbb{Z}} \hat{f}(n) x_{n}$ et on a donc $f(\tilde{T}) y_{0} \in B$.

Soit $l \in \tilde{B}^{*}$ et soit $\varphi$ l'hyperfonction $z \rightarrow\left\langle(\tilde{T}-z \tilde{I})^{-1} y_{0}, l\right\rangle(z \in \mathbb{C} \backslash \mathbb{T})$ de sorte que $\hat{\varphi}(n)=\left\langle\hat{T}^{-n} y_{0}, l\right\rangle(n \in \mathbb{Z})$. Le produit $f . \varphi$ étant défini comme plus haut on a, pour tout $n \in \mathbb{Z}$

$$
\widehat{f \cdot \varphi}(n)=\left\langle\tilde{T}^{-n} f(\tilde{T}) y_{0}, l\right\rangle
$$


et donc

$$
(f . \varphi)(z)=\left\{\begin{aligned}
\sum_{n \geq 1} \widehat{f \cdot \varphi}(n) z^{n-1}=\left\langle\sum_{n \geq 1} \tilde{T}^{-n} f(\tilde{T}) y_{0} z^{n-1}, l\right\rangle & \text { pour }|z|<1 \\
-\sum_{n \leq 0} \widehat{f \cdot \varphi}(n) z^{n-1}=\left\langle-\sum_{n \leq 0} \tilde{T}^{-n} f(\tilde{T}) y_{0} z^{n-1}, l\right\rangle & \text { pour }|z|>1
\end{aligned}\right.
$$

D'où

$$
(f . \varphi)(z)=\left\langle(\tilde{T}-z \tilde{I})^{-1} f(\tilde{T}) y_{0}, l\right\rangle(z \in \mathbb{C} \backslash \mathbb{T}) .
$$

Puisque supp $f . \varphi \subset \operatorname{supp} f \cap \operatorname{supp} \varphi \subset \Gamma \backslash \Gamma_{1}$, la fonction $z \rightarrow\left\langle(\tilde{T}-z \tilde{I})^{-1} f(\tilde{T}) y_{0}, l\right\rangle$ a un prolongement analytique à travers l'arc $\Gamma_{1}$. Ceci étant vrai pour tout $l \in \tilde{B}^{*}$, on en déduit que la fonction $z \rightarrow(\tilde{T}-z \tilde{I})^{-1} f(\tilde{T}) y_{0}$ admet alors un prolongement analytique à travers l'arc $\Gamma_{1}$. D'où $f(\tilde{T}) y_{0} \in \tilde{E}$. Comme on a déjà montré que $f(\tilde{T}) y_{0} \in B$, on voit que $f(\tilde{T}) y_{0} \in E$, ce qui entraîne que $f(\tilde{T}) y_{0}=0$ puisqu'on a supposé que $E=\{0\}$. Comme $f(\tilde{T}) \neq 0$, on voit que $\operatorname{Ker} f(\tilde{T}) \cap B$ est bien un sous-espace hyper-invariant non trivial de $T$. Ceci achève la preuve du théorème.

\section{REFERENCES}

1. G. R. Allan and T. R. Ransford, Power-dominated elements in Banach algebra, Studia Math., 94 (1989), 63-79.

2. A. Atzmon, Operators which are annihilated by analytic functions and invariant subspaces, Acta Math., 144 (1980), 27-63. $3-40$.

3. A. Atzmon, On the existence of hyperinvariant subspaces, J. Operator Theory., 11 (1984),

4. B. Beauzamy, Sous-espaces invariants de type fonctionnel dans les espaces de Banach, Acta Math., 144 (1980), 65-82. $154-182$.

5. A. Beurling, Analytic continuation across a linear boundary, Acta Math., 128 (1972),

6. A. Beurling and P. Malliavin, The Fourier transforms of measures with compact support, Acta Math., 107 (1962), 291-309.

7. J. Esterle, Quasimultipliers, representations of $H^{\infty}$, and the closed ideal problem for commutative Banach algebras, Springer Lect. Notes., 975 (1983), 66-162.

8. H. Helson, Boundedness from measure theory, linear operators and approximation, Proceedings of the conference held at Oberwolfach, August 14-22, 1971, 129-137.

9. Y. Katznelson, An introduction to harmonic analysis (Dover, New York, 1976).

10. V. Q. Phong, Semigroups with non quasianalytic growth, Studia Math., 96 (1993), 229-241.

11. J. Wermer, The existence of invariant subspaces, Duke. Math. J., 19 (1952), 615-622.

\section{UNIVERSITÉ BORDEAUX I}

LAMP, ERS0127

U.F.R. DE MATHÉmatiQues et InFORMATIQUe

351, cours de la Libération. 33405 Talence Cedex

FRANCE 